\section{Adherencia a TARGA: enfoque multi-dimensional}

\section{Adherence to HAART: multi-dimensional approach}

\section{Sr Editor:}

Leímos con interés el artículo de Varela y cols. ${ }^{1}$ sobre depresión y adherencia a terapia anti-retroviral en Chile, tema importante en el éxito de la terapia. Quisiéramos comentar lo siguiente: en primer lugar, muchos estudios coinciden en que las enfermedades psiquiátricas son las co-morbilidades que afectan en mayor medida a la adherencia terapia anti-retroviral de gran actividad (TARGA) directa o indirectamente ${ }^{2}$. En forma directa porque es la enfermedad psiquiátrica más diagnosticada en pacientes con infección por VIH e indirectamente porque es producto de otros factores de riesgo como uso de alcohol y drogas, efectos secundarios de fármacos, falta de soporte social y familiar, actitudes y creencias del paciente acerca del tratamiento ${ }^{2}$. Asimismo, se ha visto que la depresión afecta la adherencia al tratamiento de enfermedades crónicas ${ }^{3}$, por lo que la hipótesis de la asociación de depresión en adherencia a TARGA es a nuestro parecer válida.

Por otra parte, el término adherencia no es bien definido en el artículo siendo punto clave para valorar la misma. Adherencia es definida como el nivel en que la toma real de la medicación se corresponde con la prescripción de ésta ${ }^{4}$. Así, una óptima adherencia es definida como la toma de $>95 \%$ de las dosis prescritas, lo que es recomendado para una supresión virológica óptima y minimizar el grado de falla ${ }^{5}$.

La adherencia al TARGA tiene un enfoque multidimensional, por lo cual múltiples factores deben ser evaluados con el propósito de confirmar la hipótesis de la asociación de depresión y adherencia: como los relacionados al tratamiento (cantidad de pastillas, frecuencia de dosificación), a la enfermedad (recuento de CD4 y tiempo transcurrido desde el diagnóstico), personales (uso de drogas y alcohol, depresión) e interpersonales (apoyo social, estigma del VIH y la confianza con el personal de salud $)^{6,7}$.

El artículo, como todo estudio observacional, tiene el riesgo de varios sesgos. En la descripción de las características demográficas de los participantes, se observa una mayor proporción de hombres. Considerando que hay más depresión en mujeres que en hombres ${ }^{8}$, podríamos inferir que hay una subestimación de la medida de asociación. Asimismo, los modelos de regresión no consideran todos los confusores que se leen en la literatura especializada, lo cual hace difícil al lector saber si el efecto realmente es el mostrado. En relación a la medida de asociación, el uso de OR en estudios transversales con una prevalencia tan alta no es lo recomendable, ya que puede hacer que haya una sobreestimación del efecto, por lo que se recomienda el uso de ratio de prevalencias (RP) ${ }^{9}$.

Finalmente, el artículo utilizó una encuesta para evaluar si existía adherencia al tratamiento siendo este un método rápido y económico. Sin embargo, tiene una moderada especificidad y baja sensibilidad, ya que su mayor utilidad radica en casos en que la población es identificada como no adherente ${ }^{10}$. En la actualidad contamos con cuestionarios validados para evaluar adherencia a TARGA, pero la recomendación de últimos estudios es de utilizar métodos asociados, para lograr una estimación de la adherencia con la mayor exactitud posible ${ }^{11}$. Se hacen necesarios más estudios, no sólo cuantitativos, sino cualitativos o mixtos para entender la magnitud del problema de adherencia y su asociación con depresión.

\section{Referencias bibliográficas}

1.- Varela M, Galdames S. Depresión y adhesión a terapia anti-retroviral en pacientes con infección por VIH atendidos en el Hospital San Pablo de Coquimbo, Chile. Rev Chilena Infectol 2014; 31: 323-8.

2.- Chibanda D, Benjamin L, Weiss H A, Abas M. Mental, neurological, and substance use disorders in people living with HIV/AIDS in low- and middle-income countries. J Acquir Immune Defic Syndr 2014; 67 (Suppl 1): S54-67.

3.- Grenard J L, Munjas B A, Adams J L, Suttorp M, Maglione M, McGlynn E A, et al. Depression and medication adherence in the treatment of chronic diseases in the United States: a meta-analysis. J Gen Intern Med 2011; 26: 1175-82.

4.- Tejada R, Alarcón J, Velásquez C, Gutiérrez C, Loarte C, Zunt J, et al. Factores asociados a la no adherencia al tratamiento antirretroviral de gran actividad durante la gestación, periparto y postparto en mujeres VIH positivas atendidas en el Instituto Nacional Materno Perinatal, LimaPerú. Rev Peru Epidemiol 2011; 15: 01-07.

5.- Dachew B A, Tesfahunegn T B, Birhanu A M. Adherence to highly active antiretroviral therapy and associated factors among children at the University of Gondar Hospital and Gondar Poly Clinic, Northwest Ethiopia: a cross-sectional institutional based study. BMC Public Health 2014; 14: 875.

6.- Langebeek N, Gisolf E H, Reiss P, Vervoort S C, Hafsteinsdóttir T B, Richter C, et al. Predictors and correlates of adherence to combination antiretroviral therapy (ART) for chronic HIV infection: a meta-analysis. BMC Med 2014; 12: 142.

7.- Katz I T, Ryu A E, Onuegbu A G, Psaros C, Weiser S D, Bangsberg D R, et al. Impact of HIV-related stigma on treatment adherence: systematic review and meta-synthesis. J Int AIDS Soc 2013; 16 (Suppl 2): 18640

8.- Degroote S, Vogelaers D, Vermeir P, Mariman A, De Rick A, Van Der Gucht B, et al. Determinants of adherence in a cohort of Belgian HIV patients: a pilot study. Acta Clin Belg 2014; 69: 111-5. 


\section{Carta al Editor}

9.- Schiaffino A, Rodríguez M, Pasarín M, Regidor E, Borrell C, Fernández E. ¿Odds ratio o razón de proporciones? Su utilización en estudios transversales. Gac Sanit 2003; 17: 70-4.

10.- Alvis O, De Coll L, Chumbimune L, Díaz C, Díaz J, Reyes M. Factores asociados a la no adherencia al tratamiento antirretroviral de gran actividad en adultos infectados con el VIH-SIDA. An Fac Med 2009; 70: 266-72.

11.- Knobel H, Escobar I, Polo R, Ortega L, Martín-Conde M T, Casado J L, et al. Recomendaciones GESIDA/SEFH/PNS para mejorar la adherencia al tratamiento antirretroviral en el año 2004. Enferm Infecc Microbiol Clin 2005; 23: 221-31.

Sandra Vadillo, Rosalie Lópezy Jacqueline Moreno. Escuela de Medicina, Universidad de Ciencias Aplicadas, Perú.

Correspondencia a: Sandra Analí Vadillo Saravia anali1392@gmail.com 